\title{
The In Vitro Antiplasmodial Activities of Aqueous Extracts of Selected Ghanaian Herbal Plants
}

\author{
Elizabeth Cudjoe, ${ }^{1,2}$ Dickson Donu, ${ }^{1,2}$ Ruth E. Okonu, ${ }^{1,2}$ Jones A. Amponsah, ${ }^{1}$ \\ and Linda E. Amoah $\left(\mathbb{D}^{1,2}\right.$ \\ ${ }^{1}$ Noguchi Memorial Institute for Medical Research, University of Ghana, Legon, Ghana \\ ${ }^{2}$ Department of Biochemistry, Cellular and Molecular Biology, University of Ghana, Legon, Ghana \\ Correspondence should be addressed to Linda E. Amoah; lamoah@noguchi.ug.edu.gh
}

Received 10 November 2019; Accepted 30 April 2020; Published 20 May 2020

Academic Editor: Bernard Marchand

Copyright (c) 2020 Elizabeth Cudjoe et al. This is an open access article distributed under the Creative Commons Attribution License, which permits unrestricted use, distribution, and reproduction in any medium, provided the original work is properly cited.

\begin{abstract}
Background. The asexual and sexual stages (gametocytes) of Plasmodium falciparum parasites are known to respond differently to antimalarial drugs. Herbal products with extended treatment regimens and inadequate dosing information are widely used to treat malaria in Ghana. This study set out to determine the in vitro activity of selected herbal extracts on the development of asexual and sexual stage malaria parasites. Methods. The 72-hour SYBR Green 1-based in vitro drug assay was used to determine the asexual parasite growth inhibitory effects exhibited by aqueous extracts of Alchornea cordifolia, Polyalthia longifolia, Moringa oleifera, and Mangifera indica on the NF54, CamWT_C580Y, and IPC 4912 strains of Plasmodium falciparum. The effects of exposure of asexual and early-stage NF54 gametocytes to varying concentrations of the aqueous herbal extracts were assessed by microscopy after 7 days of continuous culturing in the presence of the herbal extract. Qualitative and quantitative phytochemical screening were also performed on the herbal extracts. Results. In the SYBR Green 1 assay, aqueous extracts of Alchornea cordifolia exhibited moderate $\left(\mathrm{IC}_{50}\right.$ of 5.8, 17.4, and $15.8 \mu \mathrm{g} / \mathrm{ml}$ ) and Mangifera indica exhibited low ( $\mathrm{IC}_{50}$ of 65.4 , 96.7, and $81.7 \mu \mathrm{g} / \mathrm{ml}$ ) activities against the NF54, Cam WT_C580Y, and IPC 4912 parasites, respectively, whilst Polyalthia longifolia and Moringa oleifera were inactive. Long-term treatment of NF54 parasites with $1 \mathrm{mg} / \mathrm{ml}$ of Polyalthia longifolia produced the highest densities of gametocytes and the least (56\%) inhibition of asexual parasites on Day 7. Long-term treatment of NF54 parasites with $10 \mu \mathrm{g} / \mathrm{ml}$ Alchornea cordifolia resulted in complete parasite (asexual and gametocyte) clearance on Day 7. Conclusions. Alchornea cordifolia exhibited a 'moderate' activity against the three parasites tested in the 72-hour SYBR Green 1 assay and also effectively cleared both asexual parasites and gametocytes. Long-term treatment of malaria parasites with herbal extracts mimics a treatment regimen and should be used to determine the antimalarial properties of herbal extracts.
\end{abstract}

\section{Introduction}

The recent reports of artemisinin-resistant and artemisinintolerant Plasmodium falciparum (P. falciparum) parasites call for more innovative methods to control and eventually eliminate malaria [1]. Gametocytes, the sexual transmissible forms of the malaria parasite, have been suggested to be produced at each round of asexual replication in the host [2]. Gametocytes develop through five developmental stages: early-stage (stages I, II, and III) also referred to as young gametocytes and late-stage (stages IV and V)/mature gametocytes. Gametocyte development is known to last 10 days in vivo [3]; however, in vitro, early-stage gametocytes can be distinguished from asexual parasites after 7 days of continuous culturing [4].

The first-line antimalarial treatment for uncomplicated Plasmodium falciparum malaria worldwide comprises the artemisinins, which are a fast-acting class of antimalarials [5] combined with a partner antimalarial drug such as lumefantrine and referred to as artemisinin combination therapy (ACT) [6]. Therapeutic doses of antimalarial drugs are formulated to ensure complete clearance of all infecting malaria parasites; however, drug-resistant parasites or drug-tolerant parasites have evolved that thrive under drug pressure [7]. Plasmodium falciparum parasites resistant to the artemisinin 
class of antimalarial drugs were initially reported in Cambodia [8] and have also been identified in Africa [9]. These artemisinin-resistant parasites are known to produce more gametocytes in vivo [10] and consequently enhance malaria transmission.

Consumption of herbal remedies containing extracts of one or more herbs such as Alchornea cordifolia (A. cordifolia), Polyalthia longifolia ( $P$. longifolia), Moringa oleifera (M. oleifera), and Mangifera indica (M. indica) for the treatment of malaria is high in Ghana $[4,11]$. In Ghana, the course of treatment for herbal antimalarial products range between 1 and 2 weeks [4]. Exposure of P. falciparum parasites to suboptimal drug concentrations as well as longtreatment regimens with antimalarial drugs can result in the parasites developing drug resistance $[12,13]$.

Many studies have determined the antimalarial activity of herbal extracts against the asexual disease-causing parasite using the standard 72-hour drug assay [14-18], and their activity was classified as 'good' $\left(\mathrm{IC}_{50}<10 \mu \mathrm{g} / \mathrm{ml}\right)$, 'moderate' $\left(\mathrm{IC}_{50} 10 \mu \mathrm{g} / \mathrm{ml}\right.$ to $50 \mu \mathrm{g} / \mathrm{ml}$ ), 'low' (IC $5050 \mu \mathrm{g} / \mathrm{ml}$ to $100 \mu \mathrm{g} / \mathrm{ml})$, and 'inactive' $\left(\mathrm{IC}_{50}>100 \mu \mathrm{g} / \mathrm{ml}\right.$ ) based on the concentration of the product that causes a $50 \%$ reduction in parasite prevalence $\left(\mathrm{IC}_{50}\right)$ values [19]. The effects of a longer treatment regimen on both the asexual parasite and the gametocyte have more frequently been left undetermined. In this study, the short- and long-term effects of aqueous extracts of four selected herbal plants collected from the Western Region of Ghana on malaria parasite growth in vitro were determined using artemisinin-resistant IPC_4912, the artemisinin sensitive CamWT_C580Y and the chloroquine-sensitive NF54 P. falciparum parasites.

\section{Materials and Methods}

2.1. Identification and Processing of Herbal Plants. Fresh leaves of Alchornea cordifolia (A. cordifolia), Polyalthia longifolia (P. longifolia), Moringa oleifera (M. oleifera), and Mangifera indica ( $M$. indica) were obtained from the Western Region of Ghana and sent to a botanist at the University of Ghana herbarium as well as a Research Officer (Crop Scientist) at the Centre for Plant Medicine Research (CPMR) herbarium, Mampong, Ghana, for identification although no voucher specimens were kept. The leaves were air dried and ground in a blender into a rough powder. The entire amounts of powdered A. cordifolia (21.5 g), M. indica (32.0 g), P. longifolia $(17.1 \mathrm{~g})$, and M. oleifera $(6.7 \mathrm{~g})$ leaves were individually boiled at $100^{\circ} \mathrm{C}$ in $475 \mathrm{ml}$ of $\mathrm{H}_{2} \mathrm{O}$ for one hour. The solutions were then left for 18 hours at room temperature after which they were filtered three times using a Whatman ${ }^{\mathrm{TM}} 54$ filter paper and subsequently freeze dried (lyophilized) using a Labconco $^{\mathrm{TM}}$ Freeze Dryer. Stock concentrations $(50 \mathrm{mg} / \mathrm{ml})$ of each herbal product were prepared by reconstituting $250 \mathrm{mg}$ of each freeze-dried product into $5 \mathrm{ml}$ of sterile water. The stock solutions were filter sterilized through a $0.2 \mu \mathrm{m}$ Acodisc ${ }^{\mathrm{TM}}$ filter and stored at $-20^{\circ} \mathrm{C}$ for future use.

2.2. Culturing of Plasmodium Parasites. Asexual cultures of NF54 (MRA-1000: chloroquine sensitive), CamWT_C580Y (MRA-1250: artemisinin sensitive), and IPC 4912 (MRA-
1241: artemisinin resistant) were maintained in vitro using a modified method of Zirihi et al. [17] and similar to Amoah et al. [4]. Briefly, the parasites were individually cultured at $4 \%$ hematocrit $(\mathrm{O}+$ red blood cells (RBCs)) in complete parasite media (CPM: RPMI 1640 supplemented with HEPES, L-glutamine, $\mathrm{NaHCO}_{3}$, glucose, gentamycin, and AlbuMAX II) in a T75 culture flask. The cultures were maintained in an incubator set at $37^{\circ} \mathrm{C}$ with daily medium change with $\mathrm{CPM}$ and exchange of gas (92.5\% nitrogen, 5.5\% carbon dioxide, and $2 \%$ oxygen).

Synchronized ring-stage parasites were obtained by treating a culture containing more than 5\% ring-stage parasites with a solution of $5 \%$ sorbitol. Two days ( 48 hours) after synchronization, the cultures, which were predominantly ringstage parasites were plated at $2 \%$ for the SYBR Green 1 assay or $1 \%$ parasitaemia for the long-term parasite exposure assay.

2.3. 72-Hour SYBR Green I Asexual Parasite Drug Assay. A protocol similar to that described by Quashie et al. [20] and Smilkstein et al. [21] with some revisions was used to determine the inhibitory effects of the aqueous extracts on the different $P$. falciparum parasites. Briefly, a 96-well tissue culture plate was filled with five replicates of $50 \mu \mathrm{l}$ of $20 \mathrm{mg} / \mathrm{ml}$ of two herbal extracts serially diluted 10-fold for 6 additional concentrations until $0.02 \mu \mathrm{g} / \mathrm{ml}$. The first 2 of the quintuplicate wells were supplemented with $50 \mu \mathrm{l}$ of uninfected RBC (RBCs) set at $2 \%$ hematocrit in CPM; the last 3 wells were supplemented with $50 \mu \mathrm{l}$ of infected RBC (iRBCs) set at $2 \%$ parasitemia and $2 \%$ hematocrit in CPM. The last set of duplicate wells were filled with $50 \mu \mathrm{l}$ of infected RBC (iRBCs) set at $2 \%$ hematocrit and $2 \%$ parasitaemia (ring-stage parasites) in CPM and supplemented with $50 \mu \mathrm{l}$ of artesunate $(20 \mu \mathrm{g} / \mu \mathrm{l})$ serially diluted 10 -fold for 6 concentrations with CPM until $0.2 \mathrm{ng} / \mathrm{ml}$ (schematic of plate set up in Supplementary file 1). The plate was then placed into a Modular incubating chamber and gassed for 6 minutes and then incubated for 72 hours. The plate was then wrapped in aluminum foil and stored at $-80^{\circ} \mathrm{C}$ overnight, after which the plate was thawed at room temperature. Two technical replicate plates were set up for each herbal extract. To each culturecontaining well, $100 \mu \mathrm{l}$ of buffered SYBR Green (2x SYBR Green 1 dye in $20 \mathrm{mM}$ Tris- $\mathrm{HCl}$, pH 7.5 supplemented with $5 \mathrm{mM}$ EDTA, $0.08 \%$ Triton X-100, and $0.008 \%$ saponin in PBS) was added and mixed up and down. The plate was wrapped again in aluminum foil and stored in an incubator at $37^{\circ} \mathrm{C}$ for 1 hour. Fluorescence was then read on a microplate reader at $490 \mathrm{~nm}$ excitation and $530 \mathrm{~nm}$ emission.

2.4. Long-Term Parasite Exposure Assay. This assay was replicated from Amoah et al. [4] with some minor modifications. Briefly, each stock herbal product was diluted in CPM to obtain a working solution of $200 \mu \mathrm{g} / \mathrm{ml}, 20 \mu \mathrm{g} / \mathrm{ml}$, and $2 \mu \mathrm{g} / \mathrm{ml}$. Untreated CPM (no added supplement) was used as a negative control, and $5 \mathrm{ng} / \mathrm{ml}$ artesunate was used as a positive control. One hundred microliters of the working concentrations of each herbal product and the control were added to individual wells in a 24 well plate. One hundred microliters of parasite culture at $1 \%$ parasitemia and $4 \%$ hematocrit was added to the medium-filled well of the plate 
and placed in a Modular ${ }^{\circledR}$ incubating chamber. The chamber was gassed for 6 minutes with mixed gas $(92.5 \%$ nitrogen, $5.5 \%$ carbon dioxide, and $2 \%$ oxygen) and placed in an incubator set at $37^{\circ} \mathrm{C}$. The media on the cultures in the plate were changed daily with the initial starting medium (CPM supplemented with $100 \mu \mathrm{g} / \mathrm{ml}, 10 \mu \mathrm{g} / \mathrm{ml}$, and $1 \mu \mathrm{g} / \mathrm{ml}$ of herbal extract) throughout the assay. Thin-film smears were prepared alongside the daily medium change every day for 7 days. The smears were fixed in absolute methanol and stained with $10 \%$ Giemsa for 15 minutes. Daily smears were processed and observed under a compound light microscope using a 100x oil immersion objective lens; however, only Day 7 smears were counted against 1000 RBCs for asexual parasites and 5000 RBCs for gametocytes. Each assay was conducted in duplicate and repeated at least twice.

2.5. Qualitative Phytochemical Analysis. Phytochemical screening of the individual aqueous herbal extracts was performed using previously published protocols [22-28]. The Frothing test for saponins [29]; Fehling's test for reducing sugars [29]; the Ferric chloride test for phenolic compounds, polyuronides, and cyanogenic glycosides; Mayer's test for alkaloids; Shinoda's test for flavonoids; and the Salkoski test for triterpenes and phytosterols were performed [29]. The presence of anthracenosides was also determined using a protocol described in Benmehdi et al.'s study [25]. All assays were performed with little to no modification to the published protocols and were set up in triplicate and repeated at least twice.

2.6. Quantitative Phytochemical Analysis. The total phenolic content of aqueous extracts of $A$. cordifolia and $M$. indica was determined using the Folin-Ciocalteau method [30-32]. Total flavonoid content was determined using a procedure similar to Madaan et al.'s [32], and the total saponin content was determined using a method similar to that reported by Karimi et al. [33].

All assays were done in triplicate and repeated at least twice.

2.7. Statistical Analysis. For the SYBR Green 1 drug assays, the data obtained from the herbal extract-treated uninfected $\mathrm{RBC}$ was used as the background and subtracted from the corresponding infected RBC dataset. Data was transformed, normalized, and subjected to a nonfit linear regression test ( $\log$ (inhibitor) vs. normalized response test) to enable estimation of the $50 \%$ inhibitory concentrations $\left(\mathrm{IC}_{50}\right)$ for each herbal product.

The $50 \%$ inhibitory concentration $\left(\mathrm{IC}_{50}\right)$ represents the amount of product required to kill $50 \%$ of the total parasite content of a sample.

Differences in NF54 parasite response to the 7-day treatment with the herbal products were estimated by two-way ANOVA using GraphPad Prism ${ }^{\circledR} 5.0$ software package (GraphPad Software, San Diego, CA, USA). The asexual parasitemia and gametocytaemia (early-stage gametocyte) of the continuous culture assay were obtained after counting 5000 RBC per thin smear.
TABLE 1: Yield of lyophilized aqueous herbal extract.

\begin{tabular}{lccc}
\hline Plant & $\begin{array}{c}\text { Dried ground } \\
\text { leaves }(\mathrm{g})\end{array}$ & $\begin{array}{c}\text { Lyophilized } \\
\text { extract }(\mathrm{g})\end{array}$ & $\begin{array}{c}\text { Yield } \\
(\%)\end{array}$ \\
\hline A. cordifolia & 21.5 & 2.3 & 10.7 \\
M. indica & 32.0 & 2.9 & 8.9 \\
P. longifolia & 17.1 & 1.7 & 9.7 \\
M. oleifera & 6.7 & 1.0 & 14.9 \\
\hline
\end{tabular}

The amount of lyophilized extract obtained from the dried leaves expressed as $\%$.

TABle 2: Activity $\left(\mathrm{IC}_{50}\right)$ of herbal extracts on asexual P. falciparum parasites.

\begin{tabular}{lccc}
\hline & NF54 & CamWT_C580Y & IPC 4912 \\
\hline $\begin{array}{l}\text { Artesunate } \\
(\mathrm{ng} / \mathrm{ml})\end{array}$ & $0.499 \pm 0.09$ & $0.48 \pm 0.18$ & $4.034 \pm 1.16$ \\
$\begin{array}{l}\text { A. cordifolia } \\
(\mu \mathrm{g} / \mathrm{ml})\end{array}$ & $5.81 \pm 1.34$ & $17.42 \pm 1.49$ & $15.83 \pm 1.39$ \\
$\begin{array}{l}M . \text { indica } \\
(\mu \mathrm{g} / \mathrm{ml})\end{array}$ & $65.36 \pm 1.20$ & $96.96 \pm 1.64$ & $81.68 \pm 1.31$ \\
$\begin{array}{l}P . \text { longifolia } \\
(\mu \mathrm{g} / \mathrm{ml})\end{array}$ & $161.80 \pm 0.97$ & $\mathrm{ND}$ & $\mathrm{ND}$ \\
$\begin{array}{l}M . \text { oleifera } \\
(\mu \mathrm{g} / \mathrm{ml})\end{array}$ & $176.5 \pm 0790$ & $\mathrm{ND}$ & $\mathrm{ND}$ \\
\hline
\end{tabular}

ND: not determined; $\mathrm{IC}_{50}$ : concentration at which $50 \%$ of the parasites are killed. The data is presented as the mean \pm the standard error of the mean. NF54: chloroquine-sensitive parasite; CamWT_C580Y: artemisininsensitive parasite; IPC 4912: artemisinin-resistant parasite.

The \% inhibition of the asexual parasite growth on Day 7 was calculated in Excel using the formula: (parasitemia in untreated culture - parasitemia in treated culture)/

parasitemia in untreated culture $\times 100]$.

The influence of 7 days of exposure to the herbal product on gametocyte development was calculated based on the formula: (gametocytaemia in treated cultures/gametocytaemia in untreated culture $\times 100)$.

$P$ values for statistical significance were set at 0.05 unless otherwise stated.

\section{Results}

3.1. Herbal Extract Preparation for Drug Assays. The yield of freeze-dried products from the ground leaves ranged from $9 \%$ in $M$. indica to $15 \%$ in M. oleifera (Table 1 ).

3.2. Asexual Parasite Growth Inhibition. The $50 \%$ inhibitory concentrations $\left(\mathrm{IC}_{50}\right)$ estimated for $A$. cordifolia and $M$. indica against NF54 were $5.81 \mu \mathrm{g} / \mathrm{ml}$ and $65.36 \mu \mathrm{g} / \mathrm{ml}$, respectively, and were much lower than those for $M$. oleifera and P. longifolia, which were both over $100 \mu \mathrm{g} / \mathrm{ml}$ (Table 2, Supplementary file 2). Alchornea cordifolia and $M$. indica were subsequently tested against artemisinin-sensitive (CamWT_C580Y) and artemisinin-resistant parasite (IPC 4912) isolates using the same concentration range, and both extracts Alchornia cordifolia and M. indica exhibited 'moderate' and 'low' $\mathrm{IC}_{50}$ values for both the artemisinin-resistant 


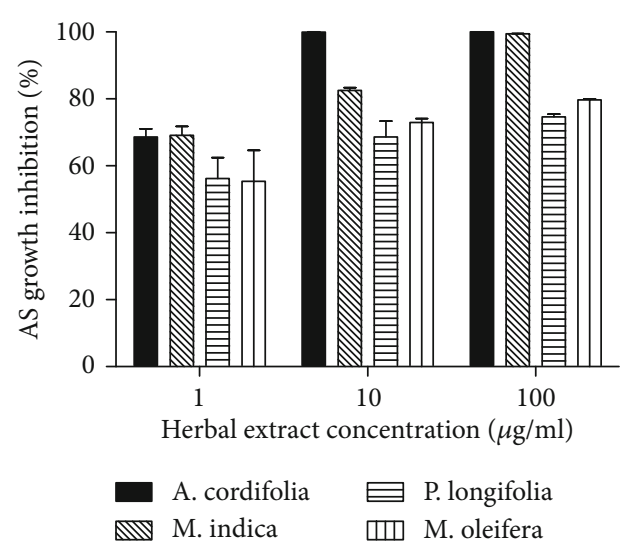

Figure 1: Herbal extract-induced asexual parasite (AS) growth inhibition. A graphical representation of the difference in the number of asexual parasites (AS; rings, trophozoites, and schizonts) identified in each thin smear expressed as a percent of the parasites identified in the control untreated culture (\% inhibition). A total number of 5000 RBCs were counted in each thin smear. The data represents the mean (SEM) of the observed growth inhibition.

and artemisinin-sensitive parasite isolates (Table 2 and Supplementary file 3). Moringa oleifera and P. longifolia, whose antimalarial activity against the NF54 parasite were in the 'inactive' category, were not further evaluated for activity against the artemisinin-sensitive and artemisininresistant parasites.

3.3. Long-Term Treatment of Asexual Parasites (AS) with the Herbal Extracts. Cultures of P. falciparum NF54 parasites treated with 1,10 , or $100 \mu \mathrm{g} / \mathrm{ml}$ of all the four herbal extracts exhibited a significant reduction $(P<0.0001$, Tukey's post hoc test, Supplementary file 4 ) in parasite density after 7 days of continuous culturing in treated media compared to parasite density in the untreated media. At $1 \mu \mathrm{g} / \mathrm{ml}$, the four herbal extract exhibited similar $(P>0.05$, Tukey's post hoc test) activity on the asexual parasite. Cultures treated with $10 \mu \mathrm{g} / \mathrm{ml}$ of A. cordifolia exhibited almost $100 \%$ parasite inhibition, which was significantly different from the inhibition exhibited by the other three extracts $(P<0.05$, Tukey's post hoc test). Cultures treated with $100 \mu \mathrm{g} / \mathrm{ml}$ of $M$. indica, resulted in $100 \%$ parasite inhibition but the cultures treated with $100 \mu \mathrm{g} / \mathrm{ml}$ of $P$. longifolia and M. oleifera reached a maximum of 75 and $80 \%$, respectively (Figure 1). Inhibition was $100 \%$ in the positive control cultures, where no parasite (asexual or gametocyte) survived after 7 days of continuous treatment in media supplemented with $5 \mathrm{ng} / \mathrm{ml}$ of artesunate.

3.4. Gametocyte Development under Constant Treatment with the Herbal Extracts. Seven days of continuous culturing of NF54 parasites in vitro with daily medium change without RBC supplementation led to differential gametocyte densities in the treated and untreated cultures (Figure 2). At $1 \mu \mathrm{g} / \mathrm{ml}$, A. cordifolia was the only product that exhibited a gametocytocidal activity; the three other products all enhanced gametocyte production. At $10 \mu \mathrm{g} / \mathrm{ml}$, A. cordifolia exhibited $99 \%$ gametocyte inhibition, which was significantly higher $(P<0.0001$, Tukey's post hoc test; Supplementary file 4$)$ than

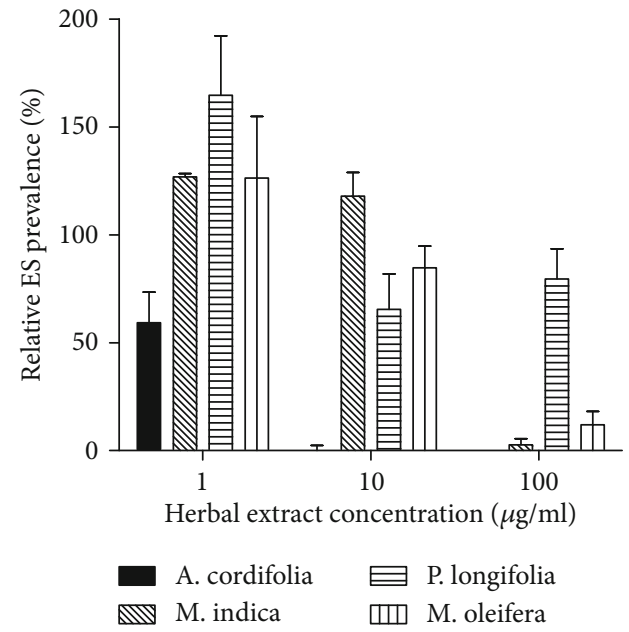

FIgURE 2: Relative prevalence of early-stage (ES) gametocytes on 7 days. A graphical representation of the total number of earlystage (ES) gametocytes (stage II) identified in each culture after 7 days of continuous exposure to herbal extracts. A total number of 5000 RBCs were counted in each thin smear, and the number of gametocytes counted was expressed as a percent of the number of gametocytes identified in the control untreated culture. The data represents the mean (SEM) number of gametocytes observed. No gametocytes were observed in the artesunate-treated cultures on Day 7.

the inhibition exhibited by similar concentrations of the other three extracts. Mangifera indica produced significantly higher numbers of gametocytes than the untreated cultures even at $10 \mu \mathrm{g} / \mathrm{ml}(P<0.05$, Tukey's post hoc test $)$. There was a general dose-response effect, with an increase in extract concentration yielding higher gametocytocidal activity and all extracts excluding P. longifolia exhibiting almost $100 \%$ gametocytocidal activity at $100 \mu \mathrm{g} / \mathrm{ml}$. No significant reduction in the prevalence of gametocytes was observed between cultures treated with $10 \mu \mathrm{g} / \mathrm{ml}$ and $100 \mu \mathrm{g} / \mathrm{ml}$ of P. longifolia.

3.5. Phytochemical Analysis. Qualitative and quantitative phytochemical analyses were conducted on A. cordifolia and $M$. indica, which both exhibited activity against the asexual parasite. Aqueous extracts of both contained reducing sugars, phenolic compounds, and flavonoids. M. indica contained saponins, which were absent in A. cordifolia (Table 3). A. cordifolia had a higher content of both total phenolics and total flavonoids than M. indica (Table 4).

\section{Discussion}

Alchornea cordifolia, M. indica, P. longifolia, and M. oleifera are components of some well-known herbal antimalarial products sold in the Ashanti Region of Ghana [34] although not prominent in herbal antimalarial products sold in the Tema metropolis of the Greater Accra Region of Ghana (Supplementary file 5). A number of studies have characterized the antimalarial properties of some herbal extracts; however, only a few studies have reported the effects that longterm administration of varying concentrations of herbal products have on the malaria parasite [4]. This study 
TABLe 3: Qualitative phytochemical analysis of A. cordifolia and $M$. indica.

\begin{tabular}{lcc}
\hline Phytochemical & A. cordifolia & M. indica \\
\hline Saponins & - & + \\
Reducing sugars & + & + \\
Phenolic compounds & + & + \\
Cyanogenic glycosides & - & - \\
Alkaloids & - & - \\
Anthracenosides & - & - \\
Triterpenes & - & - \\
Phytosterols & - & - \\
Flavonoids & + & + \\
\hline
\end{tabular}

+ indicates present; - indicates absent.

determined the $\mathrm{IC}_{50}$ values as well as evaluated the longterm effects of exposing malaria parasites to varying doses of the selected herbal extracts on artemisinin-sensitive and artemisinin-resistant malaria in order to determine whether prolonged herbal extract could enhance gametocyte production as well as access their effectiveness against artemisinin-resistant parasites.

In this study, M. oleifera was found to be inactive against the asexual NF54 P. falciparum parasite. This is similar to previous findings where methanolic extracts of $M$. oleifera exhibited low and no activity against the poW and Dd2 P. falciparum strains, respectively [35]. The inactivity of $P$. longifolia against the NF54 parasite is contrary to a recent report where an aqueous $P$. longifolia leaf extract exhibited a moderate activity against the 3D7 parasite strain [36] but supports previous studies where P. longifolia exhibited activities close to the inactive' range (between 92.6 and $100 \mu \mathrm{g} / \mathrm{ml}$ ) against the $\mathrm{K} 1$ $P$. falciparum parasite strain [37]. However, total methanolic extract of $P$. longifera was found to exhibit a 'moderate' activity $\left(\mathrm{IC}_{50} 22.04 \mu \mathrm{g} / \mathrm{ml}\right.$ ) against the $\mathrm{K} 1$ strain [38].

The $50 \%$ effective concentration $\left(\mathrm{EC}_{50}\right)$ of an extract of $A$. cordifolia fruit against the 3D7 P. falciparum parasite strain using the parasite lactate dehydrogenase assay was found to be $4.9 \mu \mathrm{g} / \mathrm{ml}$ [39] which is similar to $5.81 \mu \mathrm{g} / \mathrm{ml}$ of the NF54 strain that was obtained in this study (Table 2, Supplementary file 2). Another study in Ghana estimated the $\mathrm{IC}_{50}$ for a $50 \%$ ethanolic extract of $A$. cordifolia leaves against the 3D7 parasite to be $14 \mu \mathrm{g} / \mathrm{ml}$ using a similar SYBR Green 1 assay [40]. The main difference in $\mathrm{IC}_{50}$ values could be attributed to the solvent [41], as the active agent in A. cordifolia is ellagic acid [42], which is more soluble in water than ethanol. The aqueous extract of $A$. cordifolia exhibited a 'moderate' activity against the artemisinin-resistant parasite (Table 2, Supplementary file 3), which is an excellent indication that it has a potent antimalarial activity that could be used effectively to treat artemisinin-resistant parasites. The aqueous extract of $M$. indica exhibited a 'low' activity against the artemisinin-resistant parasite (Table 2, Supplementary file 2 ), which suggests that it would not be a good candidate to effectively treat an infection that comprises of artemisininresistant parasites. Both $A$. cordifolia and $M$. indica however exhibited a similar activity against both the artemisinin- sensitive and artemisinin-resistant parasite isolates, suggesting that their possible modes of action against the malaria parasite are different from the mode of action of artesunate, which exhibited a 10 -fold difference in 50\% inhibitory concentration against the artermisinin-sensitive and artemisinin-resistant parasites (Table 2).

After 7 days of continuous culture of parasites in the presence of herbal extracts, none of the concentrations of $P$. longifolia or M. oleifera tested were able to clear $100 \%$ of the infecting parasites (Figure 1). Combined with the fact they exhibited an activity against the malaria parasite in the 'inactive' range suggest that aqueous extracts of these two herbs do not work independently as effective antimalarial agents.

The increase in gametocyte counts (enhanced gametocyte production) in all cultures treated with $1 \mu \mathrm{g} / \mathrm{ml}$ of the herbal extracts (Figure 2) could be due to the fact that suboptimal drug treatment has been suggested to indirectly increase gametocyte prevalence [12]. Polyalthia longifolia exhibited the least gametocytocidal activity at all the tested concentrations and did not exhibit any dose response, suggesting that treatment of malaria with $P$. longifolia at concentrations of $100 \mu \mathrm{g} / \mathrm{ml}$ and below may sustain the transmission of malaria transmission due to the persistence of gametocytes after treatment. Alchornea cordifolia exhibited the highest gametocydal activity (Figure 2), even at low concentrations suggesting its potential use as a gametocytocydal agent.

A. cordifolia and $M$. indica, which exhibited 'good' and 'low' antimalarial activities, respectively, according to the classification by Batista et al. [19], were selected for qualitative phytochemical screening followed by a quantitative phytochemical screening of positive compounds identified in the qualitative screen (Tables 3 and 4). Although some previous studies have reported the phytochemical composition of these two plants, it was important to repeat the analysis on the extracts used in this study because different species of the same plant [43], as well as certain growth conditions, have been noted to influence the phytochemical content of medicinal plants. Phytochemical analysis of herbal extracts obtained from plants grown in different geographical settings reveals vast differences in phytochemical content [44], with soil from the rainforest suggested to be capable of increasing the phenol content of some plants [45].

Flavonoids and phenolic compounds in herbal plants have been associated with antimalarial activity [46] and could be the reason for the much higher activity exhibited by $A$. cordifolia, which contained much higher levels of phenolic and flavonoid content relative to M. indica (Table 4). The phenolic content of $A$. cordifolia has been suggested to be mainly composed of ellagic acid, a water-soluble polyphenol that has been suggested to be responsible for the antiplasmodial activity of A. cordifolia [47].

This study did not use the Ring Survival Assay to assess or validate the artemisinin sensitivities of the different parasites used in this study. Further studies are needed to determine whether A. cordifolia and $M$. indica extracts exhibit a gametocytocidal activity on mature gametocytes. The antimalarial activities of component fractions of A. cordifolia and M. indica also need to be investigated. 
TABLE 4: Quantitative phytochemical analysis of A. cordifolia and M. indica.

\begin{tabular}{lcc}
\hline & A. cordifolia (aq.) & M. indica (aq.) \\
\hline Total phenolics (mg GAE/g extract) & $91.5 \pm 4.0$ & $25.8 \pm 1.0$ \\
Total flavonoids (mg RE/g extract) & $0.876 \pm 0.002$ & $0.600 \pm 0.005$ \\
Total saponin content (mg DE/g extract) & ND & $1.801 \pm 0.03$ \\
\hline
\end{tabular}

ND: not detected; GAE: gallic acid equivalent; RE: rutin equivalent; DE: diosgenin equivalents.

\section{Conclusion}

Alchornea cordifolia exhibited a 'moderate' activity against the three parasites tested in the 72-hour SYBR Green 1 assay and also effectively cleared both asexual parasites and gametocytes. Long-term treatment of malaria parasites with herbal extracts mimics a treatment regimen and should be used to determine the antimalarial properties of herbal extracts.

\section{Data Availability}

The data used to support the findings of this study are included within the article.

\section{Disclosure}

The funder had no influence on the implementation of the project.

\section{Conflicts of Interest}

The authors declare that they have no conflict of interest.

\section{Authors' Contributions}

Linda E. Amoah conceived and designed the study as well as performed the statistical analysis. Elizabeth Cudjoe, Dickson Donu, Ruth E. Okonu, and Jones A. Amponsah performed the experiments.

\section{Acknowledgments}

The authors thank Frederick Tei-Maya and Benjamin Ayensu of the Department of Biochemistry, Cell and Molecular Biology, University of Ghana as well as the staff of the Department of Plant and Environmental Biology, the University of Ghana for help in performing the quantitative phytochemical analysis. We are also grateful to BEIR/MR4 for the provision of all the $P$. falciparum parasites (NF54, IPC 4912, and CamWT_C580Y) used in this study. This project was supported in part by the Book and Research Allowance from the Government of Ghana to LEA.

\section{Supplementary Materials}

Supplementary file 1: illustration of SYBR Green 1 assay plate set up. Supplementary file 2: graphical representation of NF54 asexual parasite growth inhibition. Supplementary file 3: graphical representation of IPC 4912 and CamWT_ C580Y asexual parasite growth inhibition. Supplementary file 4: statistical analysis of the D7 treated samples. Supple- mentary file 5: frequency of use of herbal extracts in 19 licensed herbal products. (Supplementary Materials)

\section{References}

[1] D. Menard and A. Dondorp, "Antimalarial drug resistance: a threat to malaria elimination," Cold Spring Harbor Perspectives in Medicine, vol. 7, no. 7, 2017.

[2] S. Eksi, B. J. Morahan, Y. Haile et al., "Plasmodium falciparum gametocyte development $1(P f g d v 1)$ and gametocytogenesis early gene identification and commitment to sexual development," PLoS Pathogens, vol. 8, no. 10, article e1002964, 2012.

[3] F. Hawking, M. E. Wilson, and K. Gammage, "Evidence for cyclic development and short-lived maturity in the gametocytes of Plasmodium falciparum," Transactions of the Royal Society of Tropical Medicine and Hygiene, vol. 65, no. 5, pp. 549-559, 1971.

[4] L. E. Amoah, C. Kakaney, B. Kwansa-Bentum, and K. A. Kusi, "Activity of herbal medicines on Plasmodium falciparum gametocytes: implications for malaria transmission in Ghana," PLoS One, vol. 10, no. 11, article e0142587, 2015.

[5] S. R. Meshnick, "Artemisinin: mechanisms of action, resistance and toxicity," International Journal for Parasitology, vol. 32, no. 13, pp. 1655-1660, 2002.

[6] The malERA Consultative Group on Drugs, "A research agenda for malaria eradication: drugs," PLoS Medicine, vol. 8, article e1000402, no. 1, 2011.

[7] G. C. Coles, "Drug resistance and drug tolerance in parasites," Trends in Parasitology, vol. 22, no. 8, p. 348, 2006.

[8] H. Noedl, Y. Se, K. Schaecher, B. L. Smith, D. Socheat, and M. M. Fukuda, "Evidence of artemisinin-resistant malaria in western Cambodia," The New England Journal of Medicine, vol. 359, no. 24, pp. 2619-2620, 2008.

[9] F. Lu, R. Culleton, and J. Cao, "Artemisinin-ResistantPlasmodium falciparumin Africa," The New England Journal of Medicine, vol. 377, no. 3, pp. 305-306, 2017.

[10] E. A. Ashley, M. Dhorda, R. M. Fairhurst et al., "Spread of artemisinin resistance in Plasmodium falciparum malaria," The New England Journal of Medicine, vol. 371, no. 5, pp. 411423, 2014.

[11] B. Graz, A. Y. Kitua, and H. M. Malebo, "To what extent can traditional medicine contribute a complementary or alternative solution to malaria control programmes?," Malaria Journal, vol. 10, no. S1, article S6, 2011.

[12] I. Petersen, R. Eastman, and M. Lanzer, "Drug-resistant malaria: molecular mechanisms and implications for public health," FEBS Letters, vol. 585, no. 11, pp. 1551-1562, 2011.

[13] K. I. Barnes, W. M. Watkins, and N. J. White, "Antimalarial dosing regimens and drug resistance," Trends in Parasitology, vol. 24, no. 3, pp. 127-134, 2008. 
[14] B. Lawal, O. K. Shittu, A. Y. Kabiru et al., "Potential antimalarials from African natural products: a reviw," Journal of Intercultural Ethnopharmacology, vol. 4, no. 4, pp. 318-343, 2015.

[15] C. van Ooil, "Combining traditional medicine and modern chemistry to fight malaria," Annals of Translational Medicine, vol. 4, no. 24, p. 550, 2016.

[16] M. do Ceu de Madureira, A. Paula Martins, M. Gomes, J. Paiva, A. Proenca da Cunha, and V. do Rosario, "Antimalarial activity of medicinal plants used in traditional medicine in S. Tomé and Príncipe islands," Journal of Ethnopharmacology, vol. 81, no. 1, pp. 23-29, 2002.

[17] G. N. Zirihi, L. Mambu, F. Guede-Guina, B. Bodo, and P. Grellier, "In vitro antiplasmodial activity and cytotoxicity of 33 West African plants used for treatment of malaria," Journal of Ethnopharmacology, vol. 98, no. 3, pp. 281-285, 2005.

[18] M. K. Laryea and L. S. Borquaye, "Antimalarial efficacy and toxicological assessment of extracts of some Ghanaian medicinal plants," Journal of Parasitology Research, vol. 2019, Article ID 1630405, 9 pages, 2019.

[19] R. Batista, J. Silva Ade Jr., and A. B. de Oliveira, "Plant-derived antimalarial agents: new leads and efficient phytomedicines. Part II. Non-alkaloidal natural products," Molecules, vol. 14, no. 8, pp. 3037-3072, 2009.

[20] N. B. Quashie, N. O. Duah, B. Abuaku et al., "A SYBR Green 1based in vitro test of susceptibility of Ghanaian Plasmodium falciparum clinical isolates to a panel of anti-malarial drugs," Malaria Journal, vol. 12, no. 1, p. 450, 2013.

[21] M. Smilkstein, N. Sriwilaijaroen, J. X. Kelly, P. Wilairat, and M. Riscoe, "Simple and inexpensive fluorescence-based technique for high-throughput antimalarial drug screening," Antimicrobial Agents and Chemotherapy, vol. 48, no. 5, pp. 18031806, 2004.

[22] J. Parekh and S. Chanda, "Antibacterial and phytochemical studies on twelve species of Indian medicinal plants," African Journal of Biomedical Research, vol. 10, pp. 175-181, 2007.

[23] C. Vimalkumar, V. Hosagaudar, S. Suja, V. Vilash, N. Krishnakumar, and P. Latha, "Comparative preliminary phytochemical analysis of ethanolic extracts of leaves of Olea dioica Roxb., infected with the rust fungus Zaghouania oleae (E.J. Butler) Cummins and non-infected plants," Journal of Pharmacognosy and Phytochemistry, vol. 3, pp. 69-72, 2014.

[24] R. Jagessar, "Phytochemical screening and chromatographic profile of the ethanolic and aqueous extract of Passiflora edulis and Vicia faba L. (Fabaceae)," Journal of Pharmacognosy and Phytochemistry, vol. 6, pp. 1714-1721, 2017.

[25] H. Benmehdi, O. Hasnaoui, O. Benali, and F. Salhi, "Phytochemical investigation of leaves and fruits extracts of Chamaerops humilis L," Journal of Materials and Environmental Science, vol. 3, p. 18, 2012.

[26] J. Senguttuvan, S. Paulsamy, and K. Karthika, "Phytochemical analysis and evaluation of leaf and root parts of the medicinal herb, Hypochaeris radicata L. for in vitro antioxidant activities," Asian Pacific Journal of Tropical Biomedicine, vol. 4, Suppl 1, pp. S359-S367, 2014.

[27] Y. Vaghasiya, R. Dave, and S. Chanda, "Phytochemical analysis of some medicinal plants from western region of India," Research Journal of Medicinal Plants, vol. 5, no. 5, pp. 567576, 2011.

[28] H. Usman, F. Abdulrahman, and A. Usman, "Qualitative phytochemical screening and in vitro antimicrobial effects of methanol stem bark extract of Ficus thonningii (Moraceae),"
African Journal of Traditional, Complementary, and Alternative Medicines, vol. 6, pp. 289-295, 2009.

[29] E. Sofowora, Phytochemical Assays, Willey \& Sons, New York, 1993.

[30] R. L. Prior, X. Wu, and K. Schaich, "Standardized methods for the determination of antioxidant capacity and phenolics in foods and dietary supplements," Journal of Agricultural and Food Chemistry, vol. 53, no. 10, pp. 4290-4302, 2005.

[31] E. A. Ainsworth and K. M. Gillespie, "Estimation of total phenolic content and other oxidation substrates in plant tissues using Folin-Ciocalteu reagent," Nature Protocols, vol. 2, no. 4, pp. 875-877, 2007.

[32] R. Madaan, G. Bansal, S. Kumar, and A. Sharma, "Estimation of total phenols and flavonoids in extracts of Actaea spicata roots and antioxidant activity studies," Indian Journal of Pharmaceutical Sciences, vol. 73, no. 6, pp. 666-669, 2011.

[33] E. Karimi, H. Z. Jaafar, and S. Ahmad, "Phytochemical analysis and antimicrobial activities of methanolic extracts of leaf, stem and root from different varieties of Labisa pumila Benth," Molecules, vol. 16, no. 6, pp. 4438-4450, 2011.

[34] G. Komlaga, C. Agyare, R. A. Dickson et al., "Medicinal plants and finished marketed herbal products used in the treatment of malaria in the Ashanti region, Ghana," Journal of Ethnopharmacology, vol. 172, pp. 333-346, 2015.

[35] I. Kohler, K. Jenett-Siems, K. Siems et al., "In vitro antiplasmodial investigation of medicinal plants from El Salvador," Zeitschrift für Naturforschung C, vol. 57, no. 3-4, pp. 277281, 2002.

[36] B. Kwansa-Bentum, K. Agyeman, J. Larbi-Akor, C. Anyigba, and R. Appiah-Opong, "In vitro assessment of antiplasmodial activity and cytotoxicity of Polyalthia longifolia leaf extracts on Plasmodium falciparum strain NF54," Malaria Research and Treatment, vol. 2019, Article ID 6976298, 9 pages, 2019.

[37] D. S. Kumari, P. V. V. Satish, K. Somaiah, N. S. Rekha, P. Barahmam, and K. Sunita, "Antimalarial activity of Polyalthia longifolia (false ashoka) against chloroquine sensitive Plasmodium falciparum 3D7 strain," World Journal of Pharmaceutical Sciences, vol. 4, pp. 495-501, 2016.

[38] S. Gbedema, Antiplasmodial evaluation of extracts of selected Ghanaian medicinal plants and other bioactivities of isolates of Polyathyia longifolia var. pendula (annonaceae), Kwame Nkrumah University of Science and Technology, Pharmaceutics, 2014.

[39] N. K. Ayisi, R. Appiah-Opong, B. Gyan, K. Bugyei, and F. Ekuban, "Plasmodium falciparum: assessment of selectivity of action of Chloroquine, Alchornea cordifolia, Ficus polita, and other drugs by a tetrazolium-based colorimetric assay," Malaria Research and Treatment, vol. 2011, Article ID 816250, 7 pages, 2011.

[40] J. Agyapong, In Vitro Assessment of Selected Ghanaian Medicinal Plants' Extracts' Activity against Plasmodium Falciparum, Kwame Nkrumah University of Science and Technology, Department of Clinical Microbiology, 2015.

[41] Q. Zhang, "Effects of extraction solvents on phytochemicals and antioxidant activities of walnut (Juglans Regia L.) green husk extracts," European Journal of Food Science and Technology, vol. 3, pp. 15-21, 2015.

[42] P. Soh, B. Witkowski, D. Olagnier, M. Nicolau, M. GarciaAlvarez, and F. Benoit-Vical, "In vitro and in vivo properties of ellagic acid in malaria treatment," Antimicrobial Agents and Chemotherapy, vol. 53, pp. 1100-1106, 2009. 
[43] S. R. Meshnick and M. J. Dobson, "The history of antimalarial drugs," in Antimalarial Chemotherapy. Infectious DiseaseHumana Press, Totowa, NJ.

[44] F. Ntie-Kang, L. L. Lifongo, C. V. Simoben, W. S. Baniaka, and L. M. Mbaze, "The uniqueness and therapeutic value of natural products from west African medicinal plants, part II: terpenoids, geographical distribution, drug discovery," RSC Advances, vol. 4, pp. 35348-35370, 2014.

[45] G. Chandler and S. Goosem, "Aspects of rainforest REGENERATION. III. The interaction of phenols, light and nutrients," The New Phytologist, vol. 92, no. 3, pp. 369-380, 1982.

[46] F. Ntie-Kang, P. A. Onguene, L. L. Lifongo, J. C. Ndom, W. Sippl, and L. M. Mbaze, "The potential of anti-malarial compounds derived from African medicinal plants, part II: a pharmacological evaluation of non-alkaloids and non-terpenoids," Malaria Journal, vol. 13, no. 1, p. 81, 2014.

[47] J. T. Banzouzi, R. Prado, H. Menan et al., "In vitro antiplasmodial activity of extracts of Alchornea cordifolia and identification of an active constituent: ellagic acid," Journal of Ethnopharmacology, vol. 81, no. 3, pp. 399-401, 2002. 